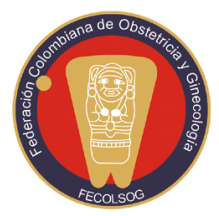

Revista Colombiana de Obstetricia y Ginecología Vol. 70 No. 1 •Enero-Marzo $2019 \bullet$ (8-18)

\title{
CAUSAS DE MUERTE MATERNA EN LA REGIÓN DE CALLAO, PERÚ. ESTUDIO DESCRIPTIVO, 2000-2015
}

\section{Causes of maternal death in the Callao region, Perú. Descriptive study, 2000-2015}

Carolina Tarqui-Mamani, $\mathbf{P h D}^{1}$; Hernán Sanabria-Rojas, $M D, \mathrm{MSc}^{2}$; Walter Portugal-Benavides, $\mathrm{MD}^{3}$; Héctor Pereyra-Zaldivar, $\mathrm{MD}, \mathrm{MSc}^{4}$; Javier Vargas-Herrera, MD, MSc ${ }^{5}$; Milena Calderón-Bedoya, Lic. enfermería ${ }^{6}$

Recibido: diciembre 29/17 - Aceptado: diciembre 21/18

\section{RESUMEN}

Objetivo: identificar las causas de mortalidad materna en la región de Callao, entre los años 2000 y 2015.

Materiales y métodos: estudio de serie de casos en establecimientos de salud (ES) públicos y privados de la región de Callao en Perú. Se incluyeron 131 mujeres como casos de muerte materna (MM) que cumplían los criterios de selección. Se conside-

1 Obstetra; doctora en Salud Pública; magíster en Epidemiología, Departamento Académico de Medicina Preventiva y Salud Pública, Facultad de Medicina Humana, Universidad Nacional Mayor de San Marcos, Lima (Perú); Instituto Nacional de Salud, Lima (Perú). ctarquim@unmsm.edu.pe

2 Médico cirujano; especialista en Enfermedades Infecciosas y Tropicales; magíster en Medicina; diplomado en Epidemiología, Departamento Académico de Medicina Preventiva y Salud Pública, Facultad de Medicina Humana, Universidad Nacional Mayor de San Marcos, Lima (Perú); Instituto Nacional de Salud, Lima (Perú). hsanabriar@unmsm.edu.pe

3 Médico cirujano; epidemiólogo. Director de Epidemiología, DIRESACallao; exdirector de la Oficina de Epidemiología de la Dirección Regional de Salud Callao, Callao (Perú). wportugalb@unmsm.edu.pe

4 Médico cirujano; pediatra; magíster en Salud Pública, Departamento Académico de Medicina Preventiva y Salud Pública, Facultad de Medicina Humana de la Universidad Nacional Mayor de San Marcos, Lima (Perú). hpereyraz@unmsm.edu.pe

5 Médico cirujano; magíster en Epidemiología, Departamento Académico de Medicina Preventiva y Salud Pública, Facultad de Medicina Humana de la Universidad Nacional Mayor de San Marcos, Lima (Perú). jvargash@unmsm.edu.pe

6 Licenciada en Enfermería; exjefa de la Unidad de Vigilancia Epidemiológica de la Dirección Regional de Salud Callao, Callao (Perú). ró $\mathrm{MM}$, mujer fallecida durante el embarazo, parto o posparto (dentro de los 42 días posparto), en ES del Callao. Se revisaron las historias clínico-epidemiológicas de MM. El análisis se realizó usando frecuencias porcentuales y promedios.

Resultados: eel 61,1\% de las causas fueron directas y el 38,9 \% indirectas. Las causas directas más frecuentes fueron los trastornos hipertensivos del embarazo, las hemorragias obstétricas y el aborto. La mediana del tiempo que tardó la gestante desde el inicio de las molestias hasta que decidió pedir atención fueron 20 minutos, la mediana del tiempo que tardó en llegar al ES luego de decidir la atención fue de 20 minutos, y la mediana del tiempo de demora desde que llegó la gestante al ES hasta ser atendida fue de 7 minutos. El 96,9\% de las muertes maternas se produjeron en ES.

Conclusión: el estudio demostró que la principal causa de MM es la directa, principalmente debido a trastornos hipertensivos del embarazo; la hemorragia obstétrica y el aborto, mientras que en menor proporción fueron las MM indirectas, principalmente enfermedades infecciosas.

Palabras clave: mortalidad materna; embarazo; epidemiología; Perú; causalidad. 


\section{ABSTRACT}

Objective: To identify the causes of maternal mortality in the Callao Region between 2000 and 2015. Materials and methods: Case series study conducted in public and private healthcare institutions in the region of Callao in Perú. Overall, 131 women who met the selection criteria were included as cases of maternal mortality (MM). MM was defined as death of a woman during pregnancy, childbirth or the postpartum period (within the first 42 days after childbirth) in healthcare institutions in $\mathrm{Ca}$ llao. MM clinical-epidemiological records were reviewed. The analysis was performed using percent frequencies and means.

Results: Of the causes of MM, 61.1\% were direct and $38.9 \%$ were indirect. The most frequent direct causes were hypertensive disorders of pregnancy, obstetric bleeding and miscarriage. Average time between the onset of discomfort and the decision to ask for assistance was 20 minutes; mean time to arrive at the healthcare institution after making the decision was 20 minutes; and mean delay time between arrival to the institution and provision of care was 7 minutes. Of the total number of maternal deaths, $96.9 \%$ occurred in a healthcare institution.

Conclusion: The study showed that the causes of MM are mainly of a direct type, primarily due to hypertensive disorders of pregnancy, obstetric bleeding and miscarriage, while indirect causes of MM were less frequent, consisting mainly of infectious causes.

Key words: Maternal mortality; pregnancy; epidemiology; Perú; causality.

\section{INTRODUCCIÓN}

La mortalidad materna (MM) es un problema de salud pública y, a la vez, un indicador del estado de salud de una comunidad. A nivel mundial, la razón de mortalidad materna (RMM) ha disminuido de 385 muertes por 100.000 nacidos vivos (NV) en 1990, a 216 por 100.000 NV en 2015 (1); en países en desarrollo para el 2015 la razón era de 239 por $100.000 \mathrm{NV}$, mientras que en los países desarro- llados era de 12 por 100.000 NV (2). En Perú, la RMM disminuyó desde 298 muertes por 100.000 nacidos vivos (NV) en el año 1990, hasta 68 por 100.000 en el año 2015 (3), lo que evidencia cierto progreso hacia la mejora de la salud materna; aun así, la RMM sigue siendo elevada si se compara con la mortalidad materna reportada en países desarrollados (4).

El quinto objetivo de Desarrollo del Milenio se relacionaba con la mejora de la salud materna, y establecía como meta la reducción de la mortalidad materna en tres cuartas partes entre 1990-2015; esta meta se cumplió (5). La mayoría de estas muertes se producen por complicaciones que pueden ser prevenibles. Al respecto, existen diversos determinantes sociales de la salud (6) asociados a la muerte materna (MM), tales como el económico, educativo, geográfico, cultural, el sistema de atención que salud que brinda la atención prenatal, parto y puerperio, entre otros (7). Es razonable tener en cuenta la importancia del acceso a los servicios de salud y la calidad de estos para reconocer signos y síntomas de alarma del embarazo, como también la capacidad resolutiva para mujeres en riesgo de MM (8).

La Organización Mundial de la Salud (OMS) señaló que las mujeres pobres tienen menos probabilidades de recibir atención sanitaria adecuada, con profesionales cualificados, y que aproximadamente solo la tercera parte de las gestantes realizan cuatro o más consultas prenatales, los partos son atendidos por profesionales de la salud y reciben atención posnatal, situación que incide en el incremento de la razón de MM (2). En la última década, el porcentaje de atención prenatal en las gestantes peruanas a nivel nacional se ha incrementado a 97,5\%, y el parto institucional a 93,0\% (9). El 97,7\% de gestantes que residen en la zona urbana tuvieron parto institucional, sin embargo, como se mencionó, la RMM sigue siendo mayor que la reportada en los países desarrollados.

La Dirección General de Epidemiología del Ministerio de Salud de Perú señala que las regiones que presentan mayor MM son Lima, La Libertad, 
Loreto, Piura y Cajamarca (10), siendo las principales causas directas la hemorragia, la hipertensión inducida por el embarazo y el aborto. La mayoría de las muertes maternas se producen en las zonas rurales, poco accesibles geográficamente y pobres, entre otras razones, hechos que reflejan la inequidad y la exclusión social de un país. No obstante, se observa que también muchas MM ocurren en la ciudad y en establecimientos de salud donde hay centros especializados para la atención del parto, con mayor capacidad resolutiva y alta cobertura del control prenatal (11).

La región de Callao, limítrofe con Lima, la capital de Perú, es fundamentalmente una zona urbana. Callao es una de las Direcciones Regionales de Salud (Diresas) que tiene mejor implementada la vigilancia epidemiológica en mortalidad materna, y emplea una ficha epidemiológica que incluye diversos aspectos personales, obstétricos, datos del embarazo, atención prenatal, parto y posparto, además de datos del establecimiento de salud, cuantifica los tiempos de demora para buscar atención, acceder a ella y recibir la atención requerida, y realiza la autopsia verbal de los casos de MM. Los casos de MM en el Callao se siguen reportando en hospitales y centros maternos públicos y privados que atienden las 24 horas del día, y son mayormente de causa directa (10). Estos establecimientos de salud, dada su cercanía con Lima, la capital, cuentan con el más alto nivel tecnológico para diagnóstico, y con personal de salud cualificado para resolver emergencias obstétricas y atención materna perinatal.

En la actualidad se han realizado diversas actividades para reducir la mortalidad materna, tales como la disminución del embarazo no deseado mediante el fortalecimiento de las acciones de promoción de la planificación familiar (12); el incremento de las atenciones prenatales tanto en la zona urbana como rural (9), lo que contribuye a mejorar la identificación de gestantes con mayor riesgo y con signos de alarma para mejorar el manejo de las complicaciones del embarazo, parto y posparto; la implementación del parto vertical en los establecimientos de salud permitiendo a la parturienta elegir la posición de dar a luz con el acompañamiento de la pareja u otro familiar (13), lo que ha incrementado la atención del parto institucional (9), y el empoderamiento de la mujer en torno a sus derechos a través de intervenciones educativas (14); sin embargo, aún se reportan muertes maternas tanto en la zona rural como en la urbana, como es el caso del Callao, en donde se cuenta con hospitales y personal especializado (10). Este panorama requiere un análisis más profundo de las causas de MM en la zona urbana, que incluya algunas características de la atención prenatal, el parto, el tiempo estimado en las demoras o retraso descrito por Deborah Maine (15), y la causa de la muerte materna (16) para proponer estrategias y acciones focalizadas que permitan disminuir las MM y consideren el contexto o escenario en donde se producen estas muertes. Por tanto, el objetivo del estudio fue identificar las causas de mortalidad materna en la región de Callao entre los años 2000-2015.

\section{MATERIALES Y MÉTODOS}

Diseño y población. Se realizó un estudio de serie de casos basado en la revisión de las fichas clínicoepidemiológicas de MM de la región de Callao. Los casos de MM fueron reportados por los hospitales y centros de salud maternos del sector público (Ministerio de Salud) que prestan atención a las gestantes no afiliadas al Seguro Social de Salud (Essalud), así como a hospitales de Essalud que brindan atención a gestantes aseguradas por el régimen contributivo de los trabajadores. Se incluyeron los casos de MM ocurridos en la región entre los años 2000 y 2015. Se excluyeron los casos de MM no obstétrica y aquellos en los que no se dispuso de la historia clínica. Se hizo muestreo consecutivo.

Procedimiento. Los casos de muerte materna fueron identificados en el registro de vigilancia de mortalidad materna de la Oficina de Epidemiología de la Dirección Regional de Salud del Callao. 
Una vez identificados, se realizó la revisión de las historias clínicas y las fichas de Investigación Epidemiológica de Muerte Materna de los casos ocurridos en el periodo de estudio. Cabe señalar que la Oficina de Epidemiología de la Diresa Callao tiene en custodia los expedientes de MM que incluyen la historia clínica, la ficha epidemiológica de MM, el análisis de los Comités de Prevención de Muerte Materna y Perinatal a nivel local y departamental, y el informe cualitativo de la MM que se realiza en el marco de la normatividad peruana (14). Se empleó una ficha ad hoc que fue validada por juicio de expertos y puesta a prueba mediante un estudio piloto. La información de las demoras en la atención fue estimada por el personal de la Diresa Callao que realizó la investigación epidemiológica de los casos de MM y que fueron registrados en la ficha de Investigación Epidemiológica de Vigilancia de MM. Una vez recolectados los datos de las fichas clínicoepidemiológicas, y hecho el control de calidad, se continuó con el procesamiento de los datos.

Definición de variables. La mortalidad materna se clasificó, según la OMS, como la muerte de la mujer durante el embarazo, parto, o dentro de los 42 días posparto, por una causa relacionada o agravada por el embarazo o su atención, pero no por causas accidentales o incidentales (9). Se consideró MM obstétrica directa como aquella producida como resultado de una complicación obstétrica del estado gravídico (embarazo, parto o puerperio), intervenciones, omisiones, tratamientos incorrectos o una cadena de eventos originada en las circunstancias mencionadas (2). La MM obstétrica indirecta es la producida como resultado de una enfermedad preexistente o de una enfermedad que apareció durante el embarazo, parto o puerperio, y que no fue debida a causas obstétricas directas, pero se agravó por los efectos fisiológicos del embarazo (17). Las MM fueron agrupadas según la Clasificación Internacional de Enfermedades (CIE-10) (17).

Adicionalmente, se midieron las características sociodemográficas (edad, ocupación, nivel de esco- laridad, estado civil), datos de la atención prenatal (control prenatal, número de controles prenatales, persona que realizó el control), datos del parto (lugar del parto, persona que atendió el parto), el plan de parto, la visita domiciliaria y signos de alarma registrados en el carné perinatal. La causa de MM se extrajo de la ficha clínico-epidemiológica que contiene la causa básica de defunción, mes, año y clasificación de MM según los protocolos de Vigilancia Epidemiológica del Ministerio de Salud de Perú (14). Se estimaron los tiempos promedio de las demoras o retrasos descritos por Deborah Maine (demora en reconocer el problema, en tomar la decisión de buscar ayuda, en llegar al establecimiento de salud y en recibir el tratamiento adecuado) (15).

Análisis estadístico. Se construyó una base de datos en SPSS v. 22, y se realizó el control de calidad correspondiente. Se evaluó la normalidad de los datos mediante la prueba de Kolmogorov Smirnov, la variable edad fue recategorizada en adolescente (10 a 19 años), joven (20 a 29 años) y adulta (30 a 49 años), y luego se calcularon frecuencias porcentuales y promedios.

Aspectos éticos. El estudio se presentó a la Dirección Regional de Salud del Callao y fue aprobado por el Comité de Ética previo a su ejecución. Se asignaron códigos a los casos de MM para asegurar la confidencialidad de los datos.

\section{RESULTADOS}

Del total de 151 registros de muertes producidas en gestantes, parturientas o puérperas, se incluyeron 131 casos de MM que cumplieron los criterios de inclusión, y se excluyeron 3 casos de muertes incidentales (asfixia, accidente de tránsito) y 17 casos (11\%) de MM porque no se encontró la historia clínico-epidemiológica (figura 1).

Entre las principales características sociodemográficas se encontró que de los 131 casos estudiados el 49,6\% [65/131] de mujeres fallecidas eran adultas, el 19,1\% [25/131] tuvieron bajo nivel de instrucción, el 51,9 \% [68/131] eran amas de casa 
Figura 1.

Distribución de muertes maternas en Callao, Perú, 2000-2015

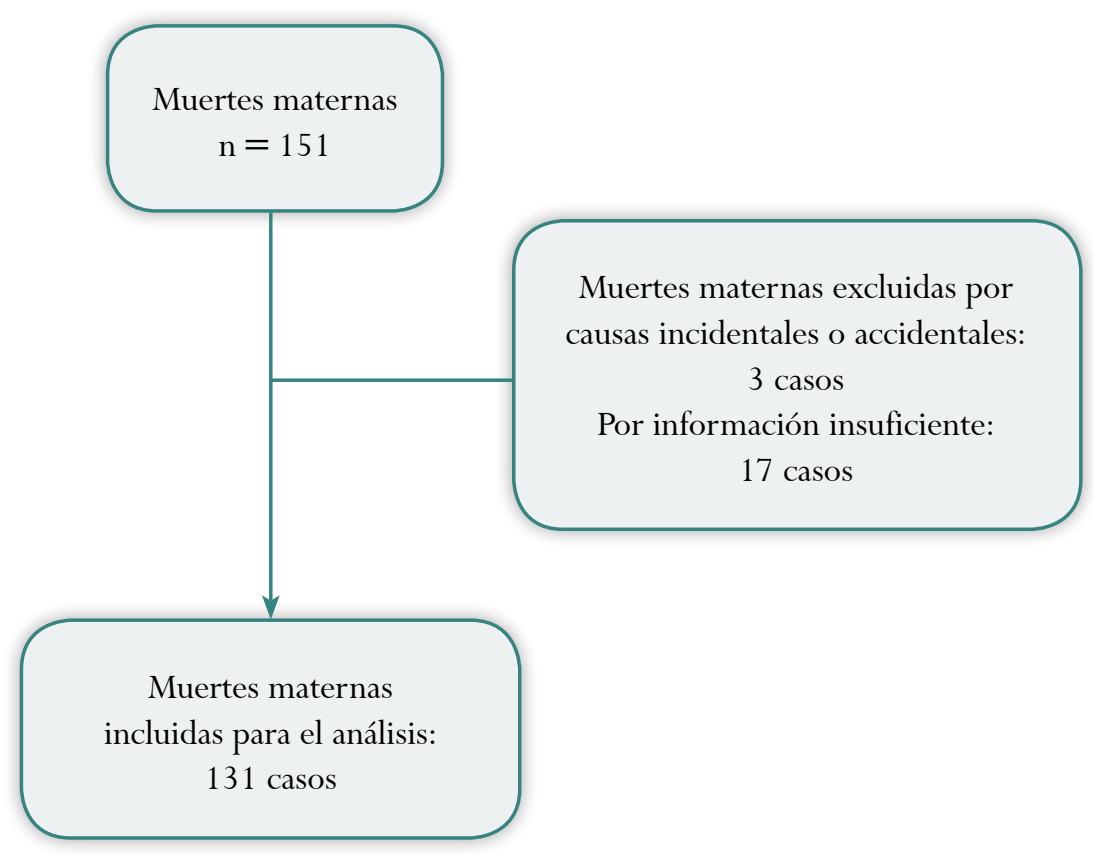

y el 68,7 \% [90/131] tenía como estado civil casada o conviviente. En la tabla 1 se presentan en detalle las características sociodemográficas.

En relación con el control prenatal, el 63,6 \% [49/77] tuvieron 4 o más controles, el 26,0 \% [20/77] tuvo escrito en el carnet prenatal los signos de alarma que se presentaron durante el embarazo (incremento de peso inadecuado, anemia, periodo intergenésico corto, hiperémesis gravídica, etc.). El 93,5\% de las gestantes no tuvieron visita domiciliaria. En la tabla 2 se presenta la distribución de otras características del control prenatal de las gestantes.

Del total de las gestantes estudiadas, el 57,3 $\%$ [75/131] reconocieron el riesgo. En el 23,7 \% [31/131] fue la propia gestante quien decidió pedir ayuda, en el 16,8 \% [22/131] fue la familia, en 9,2 $\%$ [12/131] la pareja, y se desconoce en el resto. La mediana del tiempo que tardó la gestante desde el inicio de las molestias hasta que decidió pedir atención fue de $20 \mathrm{~min}$. La mediana del tiempo que tardó la gestante para llegar al establecimiento de salud, luego de decidir pedir atención fue de $20 \mathrm{~min}$, y la mediana de tiempo desde que llegó la gestante al establecimiento de salud hasta ser atendida fue de 7 min. El 69,4 \% [93/131] de las fallecidas fueron hospitalizadas.

Según el tipo de causa obstétrica de MM, el 61,1 $\%$ [80/131] fue directa y 38,9 \% [51/131] indirecta. Entre las muertes maternas por causa directa se destacan el 32,8\% [43/131] por trastornos hipertensivo del embarazo, 12,2\% [16/131] por hemorragia obstétrica y el 10,7\% [16/131] por aborto. El 38,9\% del total de abortos fueron atendidos en el segundo nivel de atención y el 38,9\% en el tercer nivel de atención de salud. El 50,0\% de los abortos fueron atendidos por el ginecólogo, la tercera parte de estos presentó hemorragia, el 27,8\% tuvo infección. De las causas indirectas se observó que el 16,8\% fueron otras patologías (lupus eritematoso sistémico, hígado graso, aneurisma, tromboembolismo, anemia hemolítica, hemorragia digestiva, pancreatitis aguda, entre otras), 15,3\% enfermedades infecciosas 


\begin{tabular}{|c|c|c|}
\hline Características & $\mathrm{n}=131$ & $\%$ \\
\hline \multicolumn{3}{|l|}{ Edad } \\
\hline Adolescente & 12 & 9,2 \\
\hline Adulta joven & 52 & 39,7 \\
\hline Adulta & 65 & 49,6 \\
\hline Sin dato & 2 & 1,5 \\
\hline \multicolumn{3}{|l|}{ Nivel educativo } \\
\hline Analfabeto & 1 & 0,8 \\
\hline Primaria & 24 & 18,3 \\
\hline Secundaria & 60 & 45,8 \\
\hline Superior & 31 & 23,7 \\
\hline Sin dato & 15 & 11,5 \\
\hline \multicolumn{3}{|l|}{ Ocupación } \\
\hline Estudiante & 10 & 7,6 \\
\hline Ama de casa & 68 & 51,9 \\
\hline Empleada & 19 & 14,5 \\
\hline Independiente & 7 & 5,3 \\
\hline Empleada hogar & 2 & 1,5 \\
\hline Sin dato & 25 & 19,1 \\
\hline \multicolumn{3}{|l|}{ Estado civil } \\
\hline Soltera & 31 & 23,7 \\
\hline Casada/Conviviente & 90 & 68,7 \\
\hline Viuda/divorciada/separada & 2 & 1,5 \\
\hline Sin dato & 8 & 6,1 \\
\hline
\end{tabular}

*Diresa: Dirección Regional de Salud.

(tuberculosis, VIH, neumonía, bronconeumonía, AH1N1, brucelosis, estrongiloidiasis diseminada, entre otras), y 6,9\% procesos tumorales. En la tabla 3 se señalan las MM por todas las causas.

El 48,1\% [63/131] de los casos fallecieron en establecimientos de atención del Ministerio de Salud, el 43,5\% [57/131] en Essalud y el 0,8\% [1/131] en establecimientos de salud de las Fuerzas Armadas. La mayoría de las muertes maternas se produjo en establecimientos del tercer nivel de atención [86,1\%], seguidos del segundo [6,9\%] y primer nivel de atención [3,1\%]. Según los momentos del fallecimiento, la mayor parte de MM se produjo durante el puerperio [68,7\%], seguido por el embarazo [24,4\%], aborto [3,8\%], parto [2,3\%], e ignorado [0,8\%]. Hubo dos MM [1,5\%] que ocurrieron en el domicilio. Del total de MM incluidas en el estudio no se encontraron datos de necropsias en ninguna de las fichas clínicas. 


\begin{tabular}{|c|c|c|}
\hline \multicolumn{3}{|c|}{$\begin{array}{l}\text { Tabla } 2 . \\
\begin{array}{l}\text { Distribución de las características del control prenatal durante el embarazo } \\
\text { de los casos de muerte materna. Diresa* Callao, Perú, 2000-2015 }\end{array}\end{array}$} \\
\hline Características del control prenatal & $\mathrm{n}=77$ & $\%$ \\
\hline \multicolumn{3}{|l|}{ Número de cpn } \\
\hline$<1$ a 3 & 28 & 36,4 \\
\hline 4 a más & 49 & 63,6 \\
\hline \multicolumn{3}{|l|}{ Lugar de atención prenatal } \\
\hline Primer nivel & 37 & 48,1 \\
\hline Segundo nivel & 11 & 14,3 \\
\hline Tercer nivel & 17 & 22,1 \\
\hline Clínica & 1 & 1,3 \\
\hline Otro & 2 & 2,6 \\
\hline Sin dato & 9 & 11,7 \\
\hline \multicolumn{3}{|l|}{ Visita domiciliaria } \\
\hline Sí & 8 & 10,4 \\
\hline No & 69 & 89,6 \\
\hline \multicolumn{3}{|l|}{ Número de visitas domiciliarias } \\
\hline Ninguna & 72 & 93,5 \\
\hline Uno & 3 & 3,9 \\
\hline Dos & 1 & 1,3 \\
\hline Tres & 1 & 1,3 \\
\hline \multicolumn{3}{|l|}{ Especifica signos de alarma en carné prenatal } \\
\hline Sí & 20 & 26,0 \\
\hline No & 57 & 74,0 \\
\hline
\end{tabular}

${ }^{*}$ Diresa: Dirección Regional de Salud.

\section{DISCUSIÓN}

En el presente estudio se informa que en la región de Callao, Perú, la MM directa es la más importante, con la hipertensión asociada al embrazo y la hemorragia como primeras causas. En cuanto a la mortalidad indirecta, la morbilidad previa al embarazo y las infecciones no obstétricas fueron las causas más frecuentes. El análisis de las demoras mostró que los tiempos de estas son de 20 min máximo.
Nuestros resultados muestran que las causas de MM son consistentes con la distribución de muertes maternas a nivel nacional (18), lo que evidencia mayor porcentaje de MM por causa directa y menor por causa indirecta (8). Sin embargo, el porcentaje de MM por causa directa es mucho menor que el reportado a nivel nacional y en la región La Libertad en el norte peruano, donde el 81,9\% de los casos de muerte materna fueron por causa directa $(19,20)$. Al 


\begin{tabular}{|l|c|c|}
\hline \multicolumn{2}{|c|}{ Tabla 3. } & \\
\hline \multicolumn{1}{|c|}{ Distribución de las causas obstétrica de muerte materna. Diresa Callao, Perú, 2000-2015 } \\
\hline Causa de muerte materna & No $=131$ \\
\hline Directa & & 32,8 \\
\hline Trastornos hipertensivos en el embarazo & 43 & 12,2 \\
\hline Hemorragia obstétrica & 16 & 10,7 \\
\hline Aborto & 14 & 1,5 \\
\hline Infección relacionada con el embarazo & 2 & 1,5 \\
\hline Embarazo ectópico & 2 & 1,5 \\
\hline Mola hidatiforme & 2 \\
\hline Otras complicaciones obstétricas & 1 \\
\hline Indirecta & 0,8 \\
\hline Otras patologías & 22 \\
\hline Enfermedades infecciosas & 20 \\
\hline Procesos tumorales & 9 \\
\hline
\end{tabular}

*Diresa: Dirección Regional de Salud.

comparar con otros reportes internacionales de $\mathrm{MM}$ de causa directa, nuestro resultado fue mayor al $57 \%$ reportado en Baja California (México) (21), menor del 82,5\% reportado en Puebla (México), y similar a lo encontrado en el Seguro Social de México, que osciló entre 50 a $63 \%$ de MM (22).

La distribución de las causas directas más frecuentemente informadas en nuestro estudio es similar a la reportada en la región de La Libertad, en el norte peruano (20) y en todo el Perú (19), incluso en casos de MM en mexicanas atendidas en el Seguro Social (22), situación que no difiere del panorama mundial (23). El aborto constituye la tercera causa directa más frecuente en el Callao, el resultado es menor que lo observado en Ica (24) y Tacna (19), similar a lo reportado en el Perú (19) y menor que lo presentado en Chile (25); nuestros resultados son consistentes con el panorama mundial que señala que el aborto fluctúa entre el 7 al $9 \%$ en países desarrollados, países en vías de desarrollo y en América Latina y el Caribe (23). Las condiciones y el personal que realiza el aborto, sobre todo el aborto inseguro, influirían en la ocurrencia de la MM en las regiones de Ica (24) y Tacna (19). Cabe comentar que el aborto en el Perú está penalizado, por lo que existen escasos registros hospitalarios, la mayoría son registrados como hemorragia o infección (8). En ese sentido, las mujeres que deciden interrumpir su embarazo acuden a lugares clandestinos que brindan atención en precarias condiciones y con personal que podría ser no especializado, lo que aumenta el riesgo de muerte materna. Tarqui-Mamani (26) reportó una prevalencia de alrededor del $15 \%$ de la intención de interrumpir el embarazo en el Callao.

Las causas indirectas encontradas en el estudio son más altas que las informadas a nivel nacional en los años 2012 y 2015 (18, 19), y mucho mayores que

las reportadas en Argentina, donde se informó para el 2009 un incremento de la MM debido a las infecciones respiratorias, especialmente a la pandemia de influenza H1N1 (27); por otra parte, ese mismo año hubo mayor MM por el virus de la influenza en México (28).

Cerca del $9 \%$ de las muertes maternas ocurrieron en adolescentes, cifra poco menor a la reportada para MM en Chile (29). 
Sin duda, es preocupante que poco más del $40 \%$ de las gestantes no hayan tenido un control prenatal, lo cual sería un factor de riesgo para MM. Nuestra cifra de control prenatal es menor a la reportado en Perú (19), pero mayor a la presentada en un hospital nacional de Puebla (30) y Baja California en México (21). Sería conveniente analizar la calidad y el contenido de la atención prenatal. Algunos estudios señalan que las gestantes que presentan alguna complicación durante el embarazo acuden al establecimiento de salud en un estado crítico (31), o acuden a servicios de salud de baja complejidad y de primer nivel de atención que no están preparados para resolver emergencias obstétricas (32).

Nuestros resultados respecto al análisis de las demoras difieren de lo reportado en La Libertad (región del norte peruano), donde se señala que existe una gran demora en la decisión de búsqueda de atención y en llegar al ES (entre una a más de 10 horas), y menos de 10 min para ser atendidas (20), situación que podría ser explicada porque el Callao es una región totalmente urbana, accesible, cuenta con centros maternos que atienden las 24 horas, hospitales de referencia con alto nivel resolutivo y recurso humano especializado. La explicación de la mortalidad, a pesar de no haber demoras, estaría dada porque la gestante llega al ES en una situación crítica, en donde el manejo de la emergencia obstétrica puede ser insuficiente a pesar de ser atendida en menos de $10 \mathrm{~min}$. Al respecto, la normatividad peruana propone que las gestantes de alto riesgo sean atendidas por los especialistas en ginecoobstetricia, y que la referencia de las pacientes con complicaciones sea en forma oportuna y adecuada (14).

Entre las limitaciones del estudio se puede mencionar el posible sesgo de selección por no haber incluido el 11,6\% de las muertes maternas, sesgo de medición debido a que la fuente de información estuvo basada en las historias clínico-epidemiológicas de los casos que estuvieron disponibles en la Diresa Callao. Por otro lado, no se consideró el tiempo de residencia en la región de Callao, pudiendo haberse incluido algunas gestantes provenientes de otros lugares de Lima o provincias como MM registradas y notificadas por la DIRESA Callao, lo que llevaría a una sobreestimación de la frecuencia; sin embargo, hay que tener en cuenta que el Callao presenta un subregistro de 24,2\% de MM, que es discretamente mayor que el subregistro nacional (19). El diseño del estudio no permite generalizar los resultados a nivel nacional, pero aporta información para identificar las causas de MM en la región de Callao, y puede ser útil a fin de reorientar las políticas públicas para reducir la MM en la región.

\section{CONCLUSIÓN}

En la región de Callao, Perú, la principal causa de MM es la directa, principalmente debido a trastornos hipertensivos del embarazo, hemorragia obstétrica y aborto, y en menor proporción la causa indirecta, sobre todo enfermedades infecciosas.

\section{REFERENCIAS}

1. Alkema L, Chou D, Hogan D, Zhang S, Moller AB, Gemmill A, et al. Global, regional, and national levels and trends in maternal mortality between 1990 and 2015, with scenario-based projections to 2030: A systematic analysis by the UN Maternal Mortality Estimation Inter-Agency Group. The Lancet. 2016;387:462-74. https://doi.org/10.1016/S01406736(15)00838-7

2. Organización Mundial de la Salud. Mortalidad materna. Ginebra: Centro de prensa: Nota descriptiva 311; 2018. [visitado 2018 ago 20]. Disponible en: http://www.who.int/es/news-room/fact-sheets/detail/ maternal-mortality

3. WHO, UNICEF, UNFPA. The World Bank estimates. Evolución de la mortalidad materna: 1990-2015. Ginebra: World Health Organization. 2014 [visitado 2017 nov 20]. Disponible en: http://apps.who.int/iris/ bitstream/handle/10665/204114/who_rhr_15.23 spa.pdf;jsessionid $=9334513$ E95BACD3CC103D5 3880 FB5203 ? sequence $=1$ 
4. González R. Salud materno-infantil en las Américas. Rev Chil Obstet Ginecol. 2010 [visitado 2018 jul 28];75:411-21. Disponible en: https://scielo.conicyt.cl/scielo.php?script $=$ sci_arttext\&pid $=$ S0717$75262010000600011 \& \operatorname{lng}=$ es. http://dx.doi. org/10.4067/S0717-75262010000600011. https:// doi.org/10.4067/S0717-75262010000600011

5. Programa de las Naciones Unidas para el Desarrollo (PNUD). Objetivos de Desarrollo del Milenio. Informe de 2015. Nueva York: PNUD; 2015 [visitado 2018 nov 16]. Disponible en: http://www.un.org/es/ millenniumgoals/pdf/2015/mdg-report-2015_spanish.pdf

6. Organización Mundial de la Salud. Determinantes sociales de la salud en la región de las Américas. Ginebra: Centro de prensa: Nota descriptiva 311 [visitado 2018 ago 20] . Disponible en: https://www.paho.org/ salud-en-las-americas-2017/?p= 45

7. Medina-Ramírez MC, Leal-Anaya P, Aguilera-Romero TN, Leyva-Quintero E. Principales causas de mortalidad materna en Mexicali, Baja California. Ginecol Obstet Mex. 2015;83:690-6.

8. Del Carpio Ancaya L. Situación de la mortalidad maternal en el Perú, 2000-2012. Rev Peru Med Exp Salud Publica. 2013;30:461-4.

9. Instituto Nacional de Estadística e Informática (INEI). Encuesta Demográfica y de Salud Familiar 2016. Lima: INEI; 2017.

10. Dirección Regional de Salud del Callao. Situación de Vigilancia Epidemiológica de muerte materna SE. 18-2016. Callao: Dirección Regional de Salud Callao. 2016 [visitado 2017 nov 30]. Disponible en: http://www.diresacallao.gob.pe/wdiresa/documentos/ boletin/epidemiologia/DanosEnfermedades/supFILE0002442016.pdf

11. Farro A, Pacheco J. Mortalidad materna: experiencia en el Hospital Nacional Edgardo Rebagliati Martins, Essalud 1958-2002. Ginecol Obstet Perú. 2003;49:18-30.

12. Ministerio de Salud. Minsa promueve la planificación familiar para reducir la mortalidad materna. Lima: Nota de prensa; 2017 [visitado 2018 nov 18]. Disponible en: https://www.gob.pe/institucion/minsa/ noticias/13436-minsa-promueve-la-planificacionfamiliar-para-reducir-la-mortalidad-materna

13. Ministerio de Salud. Norma técnica de salud para la atención del parto vertical en el marco de los derechos humanos con pertinencia intercultural. Lima: MINSA. 2016 [visitado 2018 nov 18]. Disponible en: http:// bvs.minsa.gob.pe/local/MINSA/4240.pdf

14. Ministerio de Salud, Perú. Plan Estratégico Nacional para la Reducción de la Mortalidad Materna y Perinatal 2009-2015. Lima: Ministerio de Salud. 2009 [visitado 2017 nov 20]. Disponible en: http://www.unfpa.org. pe/publicaciones/publicacionesperu/MINSA-PENReduccion-Mortalidad-Materna2009-2015.pdf.

15. Maine D, Akalin MZ, Ward VM, Kamara A. Diseño y evaluación de programas para mortalidad materna. Nueva York: Centro para la Población y Salud Familiar, Facultad de Salud Pública, Universidad de Columbia; 1997.

16. Ministerio de Salud, Perú. Protocolos de vigilancia epidemiológica. Parte I. Lima: Oficina General de Epidemiología; 2004.

17. World Health Organization (WHO). International classification of diseases. Manual of the International Statistical Classification of Diseases, Injuries and Causes of Death. 10 ed. Geneva: WHO. 2010 [visitado 2018 ago 22]. Disponible en: www.who.int/classifications/icd/ICD10Volume2_en_2010.pdf

18. Gil F. Situación epidemiológica de la muerte materna en el Perú 2015. Boletín Epidemiológico (MINSA). 2015;25:66-74.

19. Maguiña M, Miranda J. La mortalidad materna en el Perú 2002-2011. Lima: Dirección General de Epidemiología/Ministerio de Salud. 2013 [visitado 2017 nov 6]. Disponible en: http:// www.dge.gob.pe/portal/docs/tools/MORTALIDAD_\%20MATERNAPERU.pdf.

20. Santos L, Mori L, González M. Mortalidad materna: factores determinantes modificables mediante políticas públicas en la región La Libertad. Ucv-Scientia. 2010;2(1):22-33.

21. Medina-Ramírez MC, Leal-Anaya P, Aguilera-Romero TN, Leyva-Quintero E. Principales causas de mortalidad materna en Mexicali, Baja California. Ginecol Obstet Mex. 2015;83:690-6. 
22. Ruiz R, Cruz P. Causas de mortalidad materna en el Instituto Mexicano del Seguro Social, periodo 2009-2012. Rev Med Inst Mex Seguro Soc. 2014;52:388-96.

23. Say L, Chou D, Gemmill A, Tunçalp Ö, Moller AB, Daniels J, et al. Global causes of maternal death: A WHO systematic analysis. The Lancet Global Health. 2014;2:e323-e33. https://doi. org/10.1016/S2214109X(14)70227 $\neg X$

24. Ybaseta-Medina J. Factores de riesgo asociados a mortalidad materna en la región de Ica, Perú, 2001-2005. Rev Med Panacea. 2011;1.

25. Donoso E, Vera C. Mayor mortalidad materna por aborto en Cuba que en Chile, 2000-2015. Rev Chil Obstet Ginecol. 2018 [visitado 2018 nov 27];83(3):240-9. Disponible en: https://scielo.conicyt. cl/scielo.php?script $=$ sci_arttext\&pid $=$ S07 17 $75262018000300240 \& \operatorname{lng}=$ es. https://doi. org/10.4067/s0717-75262018000300240

26. Tarqui-Mamani C, Barreda A, Barreda M, SanabriaRojas H. Prevalencia del intento de interrumpir el embarazo y factores asociados en una comunidad urbano marginal de Lima-Perú, 2006. Rev Peru Med Exp Salud Publica. 2010;27:38-44. https://doi. org/10.17843/rpmesp.2010.271.1442
27. Ministerio de la Salud. Presidencia de la República. Natalidad y mortalidad 2014: Síntesis Estadística I/2016. Buenos Aires: Dirección de Estadísticas e Información de Salud. 2016 [visitado 2017 nov 10]. Disponible en: http://www.deis.msal.gov.ar/wp-content/ uploads/2016/05/Sintesis-estadistica-Nro1.pdf.

28. Fajardo-Dolci G, Meljem-Moctezuma J, González V, Venegas E, Vicente F, Villalba-Espinoza I, et al. Análisis de las muertes maternas en México ocurridas durante 2009. Rev Med Inst Mex Seguro Soc. 2013;51:486-95.

29. Donoso E, Carvajal JA, Vera C, Poblete JA. La edad de la mujer como factor de riesgo de mortalidad materna, fetal, neonatal e infantil. Rev Med Chil. 2014;142:168-74. https://doi.org/10.4067/S003498872014000200004

30. Hernández J, López J, Ramos G, López A. Análisis de casos de muerte materna ocurridos en un periodo de 10 años. Ginecol Obstet Mex. 2007;75:61-7.

31. Gonzaga-Soriano MR, Zonana-Nacach A, AnzaldoCampos MC, Olazarán-Gutiérrez A. Atención prenatal y mortalidad materna hospitalaria en Tijuana, Baja California. Salud Publica Mex. 2014;56:32-9. https:// doi.org/10.21149/spm.v56i1.7320

32. Cáceres-Manrique F. El control prenatal: una reflexión urgente. Rev Colomb Obstet Ginecol. 2009;60:165-70. 